\section{INFLUENCING FACTORS OF LONG-TERM ABSENTEEISM: A CROSS-SECTIONAL STUDY AMONG BELGIAN EMPLOYEES}

\begin{abstract}
1,2M Verbrugghe*, 'I Vandevelde, ${ }^{1} \mathrm{~T}$ Deburghgraeve, ${ }^{1} \mathrm{I}$ Peeters, ${ }^{1} \mathrm{M}-\mathrm{N}$ Schmickler, ${ }^{3}$ B Teuwen. ${ }^{1}$ Mensura Occupational Health Services, Belgium; ${ }^{2}$ Department of Public Health, University of Ghent, Belgium; ${ }^{3}$ Certimed, Belgium
\end{abstract}

\subsection{6/oemed-2018-ICOHabstracts.502}

Introduction The increase in long-term ( $>1$ month) absenteeism is a major challenge for Belgian companies. Over the last 10 years, long-term absenteeism increased from $3.1 \%$ to $5.13 \%$. Consequently, absenteeism has become a key element in the human resource management strategy of many organisations. The aim of this study was to assess factors influencing long-term absenteeism among employees.

Methods A questionnaire was developed by a team of experts in the field of occupational health, to assess factors influencing long-term absenteeism. The questionnaire comprised 28 items covering four main topics: employee characteristics (e.g., age), absenteeism (e.g., absenteeism rate, causes of absenteeism), work-related features (e.g., employment satisfaction, stressors, social support, job control), and lifestyle (e.g., smoking). Employees were invited to fill out the questionnaire online by their employee organisations in Belgium.

Results From January to March 2017, a total of 1913 employees $(50.2 \%$ male, $49.8 \%$ female) filled out the questionnaire. The survey revealed that over the last year, about one out of five of the respondents had been on unscheduled sick leave for at least two weeks. Almost $10 \%$ of the respondents considered themselves at risk for sick-leave $\geq 1$ month during the next year. Of these, 53\% believed that physical reasons would be the cause for the absence, and $38 \%$ cited psychological reasons.

The following variables were found to be positively associated $(\mathrm{p}<0.05)$ with long-term absenteeism: type of work (physical work), seniority at work, and low social support from family and friends. On the other hand, job satisfaction and a healthy lifestyle were found to be negatively associated $(p<0.05)$ with long-term absenteeism.

Discussion The results of this study present insights into factors associated with the risk of long-term absenteeism. They provide a starting point for actions both at an organisational and individual level targeted at reducing and preventing longterm absenteeism.

\section{WHAT ARE THE MOST IMPORTANT QUESTIONS TO ANSWER? - EXPERIENCES IN PRIORITY SETTING IN COCHRANE WORK REVIEW GROUP}

Jani Ruotsalainen*, Jos Verbeek. Cochrane Work, Finnish Institute of Occupational Health, Kuopio, Finland

\subsection{6/oemed-2018-ICOHabstracts.503}

Introduction Conducting Cochrane reviews and updating them regularly requires a considerable investment from authors and the editorial base alike. It is therefore important to focus on those topics for which we think reviews are most important. Therefore, we wanted to prioritise the review titles that we have in our editorial process.

Methods In the spring of 2016, we asked our editorial board members to rank all 55 titles of Cochrane Work reviews according to how important they think each title is. The editors could choose one of five response options for each title:

1. Highest priority (invest editorial resources in updating),

2. Quite important (encourage authors to update),

3. Good topic (do not actively seek update),

4. Not really that important (never update), and

5. Lowest priority (consider deregistering).

Responses were weighted so that option 1 got three points, 2 got two points, 3 got one point and 4 as well as 5 got zero points.

Result Fifteen Cochrane Work editors participated in this survey. Interestingly, the spread of votes was very even. The titles: 'Adaptation of shift work schedules for preventing and treating sleepiness and sleep disturbances caused by shift work' and 'Interventions to prevent injuries in construction workers' were ranked as the most important, both with a mean score of 2.27 (SD 3.08).

Discussion Priority setting for systematic review topics is complex. Cochrane Work review group editors judged prevalence, impact on patient/worker, impact on employer/society, costs, and relevance for their own jurisdiction to be important reasons to assign high priority. However, these factors varied across assessors leading to little variation in priorities.

\section{HEALTH CHECKS AT THE WORKPLACE}

Stefan Webendoerfer, Matthias Claus, Christoph Oberlinner. Basf SE, Ludwigshafen, Germany

\subsection{6/oemed-2018-ICOHabstracts.504}

BASF SE in Ludwigshafen has approximately 36000 employees who receive occupational medical support from the Corporate Health Management Department. As part of demography management health checks for early recognition of chronic diseases were introduced for all employees in 2011 in addition to obligatory occupational medical prevention. Regardless the risk potential at the workstation each employee has the opportunity to make use of a health check every three years. Depending on the diagnosis, a medical recommendation is given to visit an external consulting physician or to take part in health promoting activities offered within the company. A computerised invitation procedure similar to that used in cancer prevention makes sure that entitled employees use the health check regularly.

Methods The BASF health check includes an extensive laboratory test, various technical tests such as spirometry, ECG, audiometry and eye test. Work ability index, WAI, psychological stress, pre-existing conditions and the risk profile for diabetes (Find Risk questionnaire) and heart attacks (Procam score) are captured in a questionnaire. Module recommendations on the topics of 'nutrition and exercise', 'daily strain and strain caused by stress', 'musculoskeletal disorders' and 'smoking cessation' are given based on a stored algorithm. Furthermore, individual reports about the results are elaborated by the occupational health physicians. If severe conditions at an early stage are recognised, recommendations are given to visit a primary care physician.

Results From November 1, 2011 until December 31, 2014 18140 employees made use of the offer of a health check (a participation rate of $45.3 \%)$. In accordance with the gender distribution within BASF $80.3 \%$ of the male employees and 
$19.7 \%$ of the female employees participated. The average age among the men was 45 , and 39 among the women. $42 \%$ of industrial employees, $36 \%$ of employees under a collective agreement and $21 \%$ of employees not under a collective agreement participated. In recent diagnoses musculoskeletal conditions were leading. The BMI becomes higher with increasing age, showing $23.2 \%$ of employees older than 50 a value of over 30 . Accordingly, in $20.4 \%$ of employees older than 50 a prediabetes with an HbA1C between $5.7 \%$ and $6.4 \%$ could be determined. The percentage of diabetics with an $\mathrm{HbA} 1 \mathrm{C}$ value over or equal to $6.5 \%$ was at $4.3 \%$. Module recommendations with respect to health promoting measures were given in $60 \%$ of the cases.

Discussion Almost 50\% of the employees well accept health checks for early recognition of chronic and lifestyle conditions. The proportion of 30 to 60 year olds and the proportion of men were the highest. In comparison to external health checks especially industrial employees are reached, being newly diagnosed especially diabetes mellitus, hypertension and skin diseases. Due to the standardised examination procedure the psychological stress situation can be better assessed. $18 \%$ of the participants state that they have a medium WAI while $4 \%$ have a bad WAI. The health check was well accepted at BASF and can prospectively be well evaluated relating to its effectiveness.

\section{HEALTH MANAGEMENT AT UNILEVER - RETURN ON INVESTMENT: THE UNILEVER SINGAPORE LAMPLIGHTER PROGRAM (2009 - 2015)}

N Guharajan. Unilever Asia Pte Ltd, Singapore

\subsection{6/oemed-2018-ICOHabstracts.505}

Introduction Lamplighter is Unilever's global health, well-being and performance program. Our vision is a culture in which employees and their families actively seek to embrace health and wellbeing. Lamplighter is an organisational well-being program designed to improve the health, wellbeing and performance of Unilever employees over a six to twelve month period by focusing on three main areas; exercise, nutrition and mental resilience. Led by the Medical and Occupational Health division, the Lamplighter program is fully integrated into Unilever's multi-national, multi-local activities to ensure that Health and Wellbeing initiatives reach across the business. Methods Using a Unilever designed Health Risk Questionnaire, information is collected on the prevalence of specific health risk factors. A 3rd party administered ROI (Return on Investment) tool that can measure medical and productivity risk factor costs coupled with risk prevalence of specific health risk factors, overall program costs and employee median annual compensation is used to generate the ROI. Four specific outputs are demonstrated: medical only, absenteeism only, presentism only, and overall ROI (medical and productivity combined).

Results 6 Year Aggregate (2009 through 2015): Unilever-Singapore healthcare cost return-on-investment (ROI) for the participant sample over the span of 6 periods was found to be 1.72: 1. When looking at the ROI for productivity the ratio is 0.48: 1 for absenteeism, and 1.30: 1 for presentism. When healthcare and productivity savings are combined the ratio is 3.50: 1 .

Discussion The Unilever Singapore program came into existence in 2009. Since then we have been able to demonstrate reduction in the prevalence of health risk factors while also showing a positive Return on Investment over the 6 year period. The findings indicate that our program is heading in the right direction and showing a positive impact on employee health and related health costs.

\section{IMPACT OF TARGETED INTERVENTION PROGRAMS WITH A HOLISTIC APPROACH TO IMPROVE THE HEALTH RISK PARAMETERS OF INDIAN WORKERS}

${ }^{1}$ Bishwadeep Paul ${ }^{*},{ }^{2}$ Chaitanya Gulvady. ${ }^{1}$ Siemens Ltd, Mumbai, India; ${ }^{2}$ Indian Association of Occupational Health

\subsection{6/oemed-2018-ICOHabstracts.506}

Introduction Increasing incomes and sedentary lifestyles have led to changes in food habits resulting in increasing trends of obesity and NCD's among workers. As simple disease awareness programs seemed ineffective in bringing about lifestyle modifications, a holistic approach to health was conceptualised by the Siemens India Health Management Team to improve the health risk parameters of workers and prevent future complications.

Methods Targeted intervention programs over 3 month duration each have been initiated since October 2015. The first FIT4LIFE Program had 50 identified Pre-Diabetic employees who had access to weekly Dietician consultation; Yoga sessions with emphasis on relaxation techniques and short modules for daily practice; and, daily walking 10000 steps with a mobile Pedometer App. Stress Management sessions by EAP counsellors helped increase psychosocial health awareness and its connexion to lifestyle disorders. Mobile phone application was effectively used to provide motivation and positive reinforcement to the group. Baseline and final assessment data were analysed using paired t-test.

Results At the end of the first program, participants categorised under high risk for diabetes were found to have reduced their weight and a $26 \%$ reduction of HbA1c levels was achieved. It was demonstrated that $20 \%$ participants achieved weight loss between $5 \mathrm{~kg}$ to $9.6 \mathrm{~kg}$ and $30 \%$ participants achieved weight loss between $2 \mathrm{~kg}$ to $4.9 \mathrm{~kg}$. During Final Assessment, all except 2 participants demonstrated HbA1c reduction as compared to baseline assessment stage.

Paired t-test was applied and $\mathrm{P}$ value was found to be statistically significant for 6 of the 8 parameters applied to the first group of 42 who completed the 3 month program.

Discussion The positive findings and success of the first FIT4LIFE Program has motivated the Siemens India Health Management Team to replicate the Program across its multiple locations covering over 500 workers in the last 18 months, thus creating a sustainable positive health impact

\section{PHYSICAL FITNESS ASSESSMENT FOR PREVENTING ACCIDENT AT WORK IN ENERGY INDUSTRY WORKERS}

${ }^{1}$ Penpatra Sripaiboonkij, ${ }^{2}$ Sasitorn Taptagaporn. 'School of Public Health, Physiotherapy, and Sports Science. University College Dublin, Dublin, Ireland; ' $F a c u l t y$ of Public Health, Thammasat University, Bangkok, Thailand

\subsection{6/oemed-2018-ICOHabstracts.507}

Introduction Physical fitness could be used to predict the prevalence of accident at work. It could be suggested that healthy employees suffer fewer accidents. The objective was to assess 